\title{
Customized visualizations for audience-oriented communication in COVID-19 tracking story
}

\author{
Liuhuaying Yang* \\ Hui Cheng Ang ${ }^{\dagger}$ \\ Chinese Media Group \\ Singapore Press Holdings Limited
}

Shuyang Wang

\section{INTRODUCTION}

This visual case study was conducted by zaobao.sg, the digital platform of the Chinese dailies in Singapore. In early February, to inform and communicate with local audiences about the COVID-19 situation in Singapore, we built an interactive webpage titled "Dynamic tracking of COVID-19 in Singapore" (see https://interactive.zaobao.com/2020/novel-coronavirus-casesin-singapore/ ), to visualize the daily situation reports released by the Ministry of Health. As the situation evolved, we kept updating the data every day until June 2nd, when Singapore ended its Circuit Breaker measures. The aid of the visualisation is to help better picture the relationship between cases within a cluster, how these clusters are inter-related and activities-based, and how cases are disseminated to various hospitals.

The Singapore government was among the few globally that released substantial details of the new cases. This provided us with an opportunity to explore communicative visualizations in different stages of the epidemic situation and with scales of data.

This also highlights the need for customized visualizations in journalism and beyond and underlines the importance for the newsrooms to adapt accordingly [2-5].

\section{THE DYNAMIC TRACKING WEBSITE}

Having noticed that the audience's points of interest were changing over time, we started adjusting the content and visualizations. We built multiple customized data visualizations such as the visual journey of each case, the length of hospitalization and cluster tracking.

In the initial stage, daily new cases remained largely in the tens. Instead of the overview trend typically represented by a line chart, local audiences were more interested in the details of each case compiled in the official reports. We constructed a journey visualization (see Fig. 1a) to show the key dates such as confirmed date, quarantine date and the audience can click to expand the journey and read more information such as the dates of visits to clinics, and related news.

As the number of discharged cases increased, the audience became more interested in the length and place of hospital stays. We built a two-side bar chart (see Fig. 1b) to visualize for each case the number of days between the onset of symptoms and being confirmed and the length of subsequent stay in hospital. It also allows the audience to sort by case id or the number of days, group by the hospital, gender, country of origin or age group, and filter by a particular cluster or group. This visualization intends to facilitate answering questions like whether cases of a certain age group are more vulnerable, and whether some hospitals can handle better, etc.

\footnotetext{
*e-mail: yanglhy@sph.com.sg

†e-mail: hcang@sph.com.sg

‡e-mail: shuyang@sph.com.sg
}
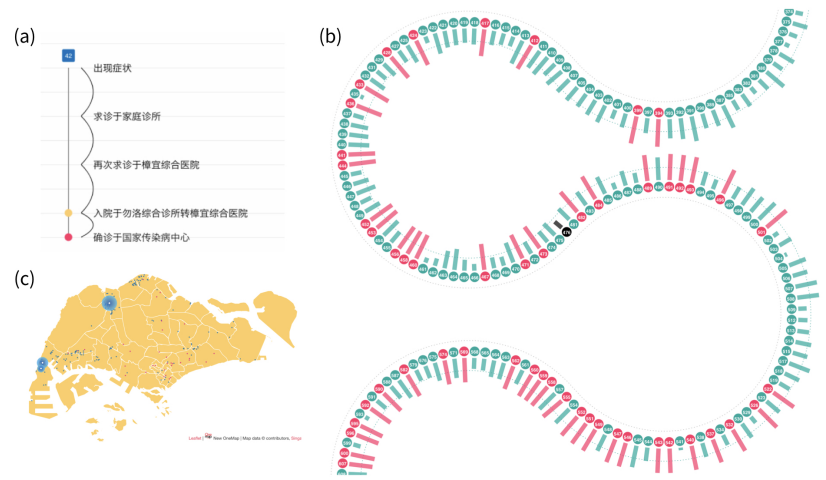

Figure 1: Examples of visualizations created in this project

However, with the number of new cases crossing 500 per day, the government stopped giving details of cases. We implemented a new visualization for summarizing the median length of known cases. The previous visualization is better presented in one of the older versions (see https://interactive.zaobao.com/2020/novel-coronaviruscases-in-singapore-v1/)

We also built a cluster tracking map (see Fig. 1c) to highlight the newly discovered clusters, and visualize the geographical distribution of the clusters by plotting clusters as tiny dots on the local map.

\section{Outcome}

This dynamic tracking webpage is the most popular interactive news and data visualization story in Zaobao's interactive news section, as reflected by the number of views in Google Analytics.

Compared with daily breaking news or news archives, the accumulated data allows us to present more comprehensive insights on both temporal (i.e. the trend) and spatial dimensions (i.e. the cluster tracking on the map). Digital interactive format provides a more engaging and exploratory experience for audiences, as the audience can use filters and search to navigate the site according to their own interests. It also served as an additional tool for journalists to study and explore potential news angles.

This study exposes both data literacy and visualization literacy issues. In terms of data literacy, the audience might fail to know that the answer they are looking for can be answered by playing with filters. We found that the audience differs in their capacity for navigating and understanding the visualization. For instance, they might fail or are reluctant to read a visualization that is not a bar chart or line chart; they might get used to thinking a visualization is always static as print and fail to hover or click. These issues not only require design considerations, but also the data visualization education for journalists who produce the content. 


\section{REFERENCES}

[1] Paul Kahn (2020). Data Visualization During the COVID-19 Pandemic. Namahn, https://namahn. com/lectures/paul-kahn-2/.

[2] Dong E, Du H, Gardner L (2020). An interactive web-based dashboard to track COVID-19 in real time. Lancet Infect Dis 20 (5), 533-534, https: //doi.org/10.1016/S1473-3099(20)30120-1.

[3] Kevin Maher (2020). Lessons From China's COVID-19 Visualizations. Nightingale, https://medium.com/nightingale/ lessons-from-chinas-epidemic-visualization-f3b25c136d51.

[4] Jason Forrest (2020). How John Burn-Murdoch's Influential Dataviz Helped The World Understand Coronavirus. Nightingale, https: //medium. com/ nightingale/how-john-burn-murdochs-influential-dataviz-helped-the-world-understand-coronavirus-6cb4a09795ae

[5] Ben Shneiderman (2020). Data Visualization's Breakthrough Moment in the COVID-19 Crisis. Nightingale, https://medium. com/nightingale/ data-visualizations-breakthrough-moment-in-the-covid-19-crisis-ce46627c7db5

\section{APPENDIX: ENGLISH EXPLANATION OF THE MAIN VISUALIZATIONS}

\section{1: Map of clusters}

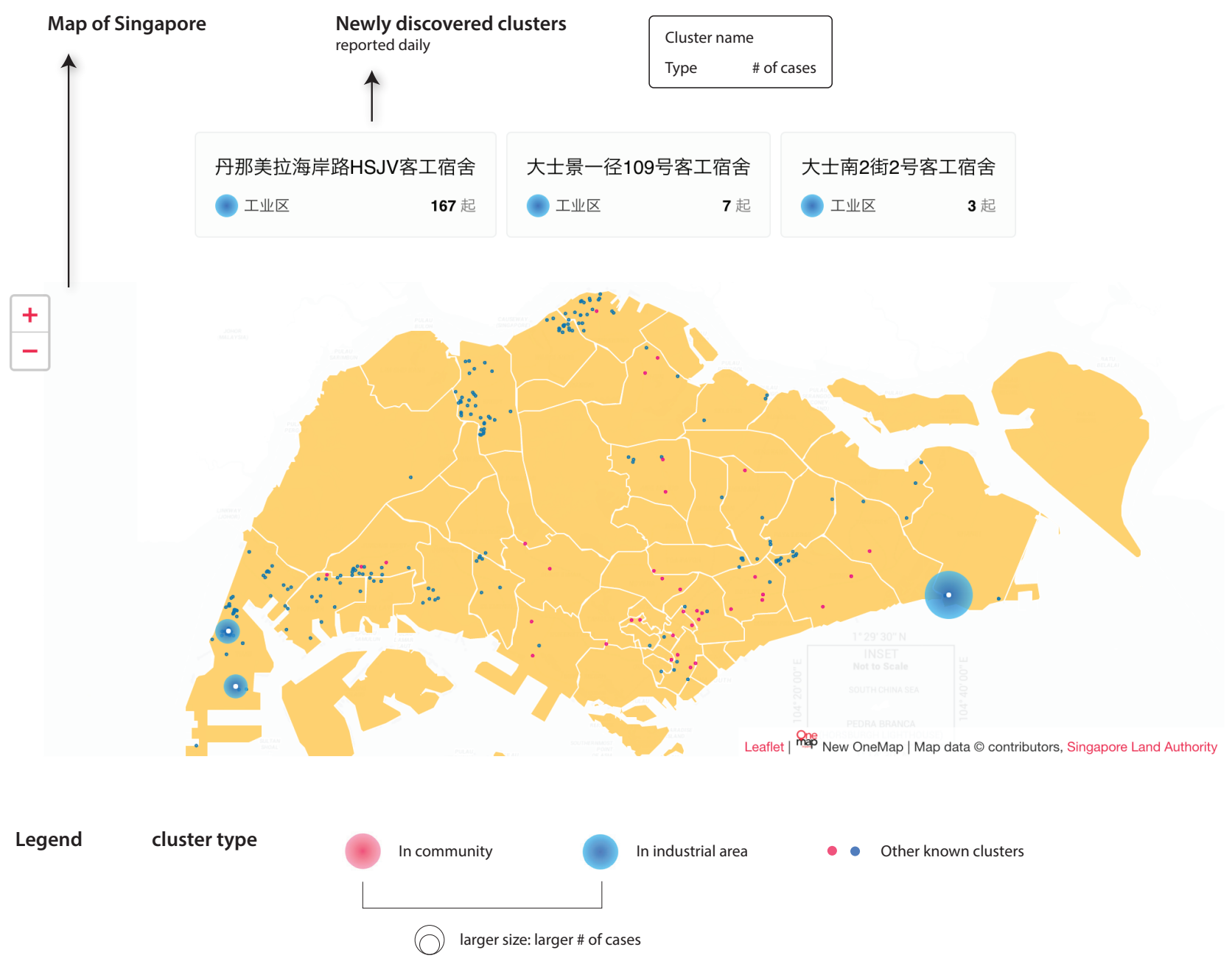




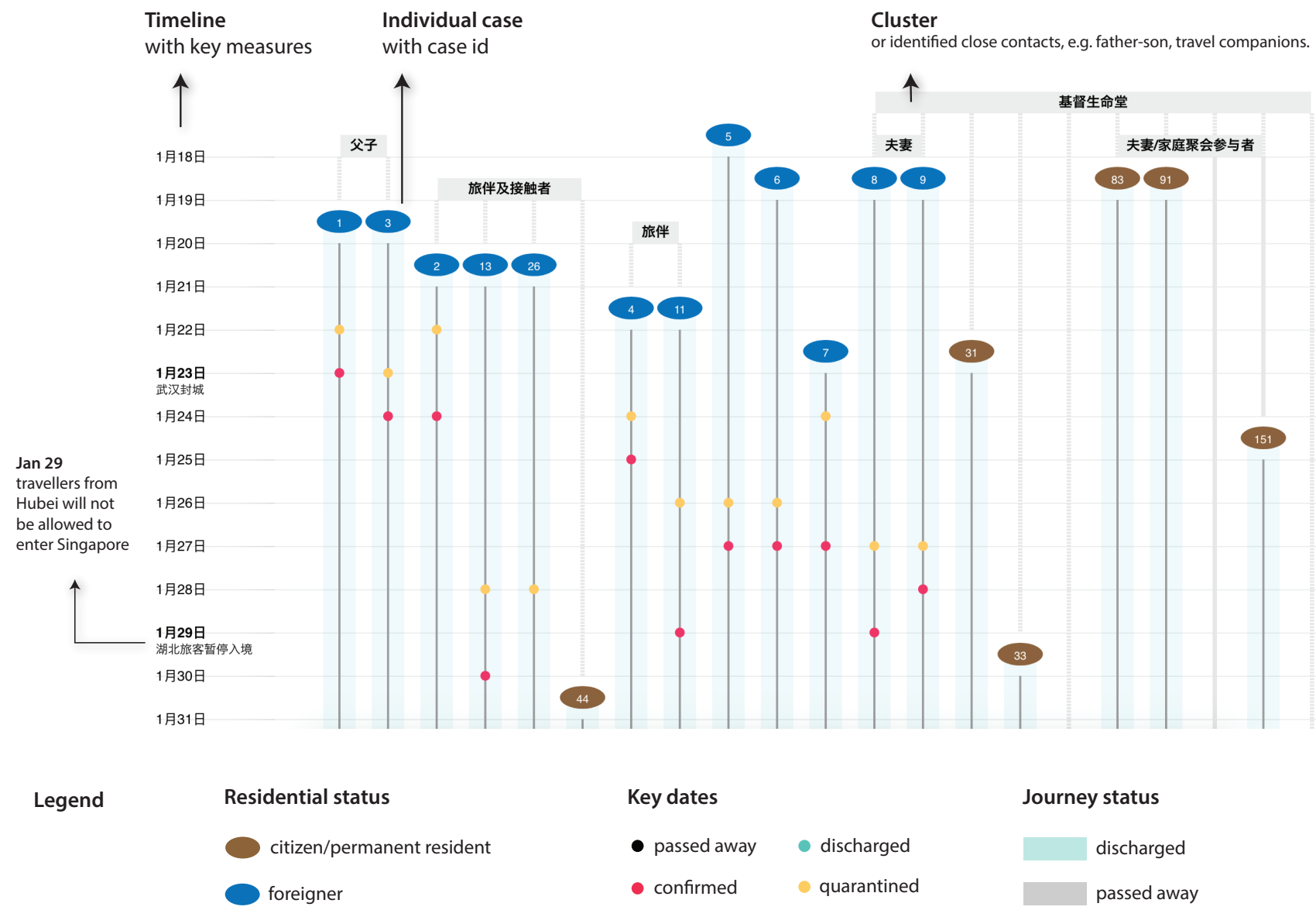

The expanded journey path after clicking

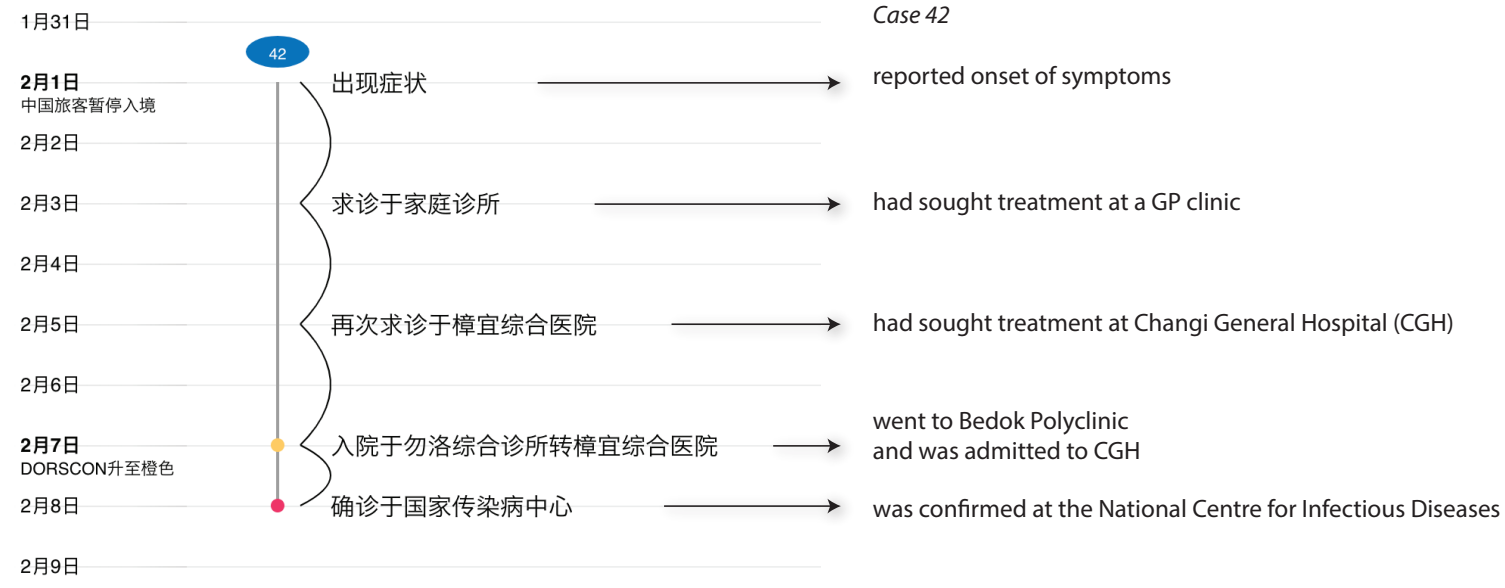


3: Visualization of discharged cases
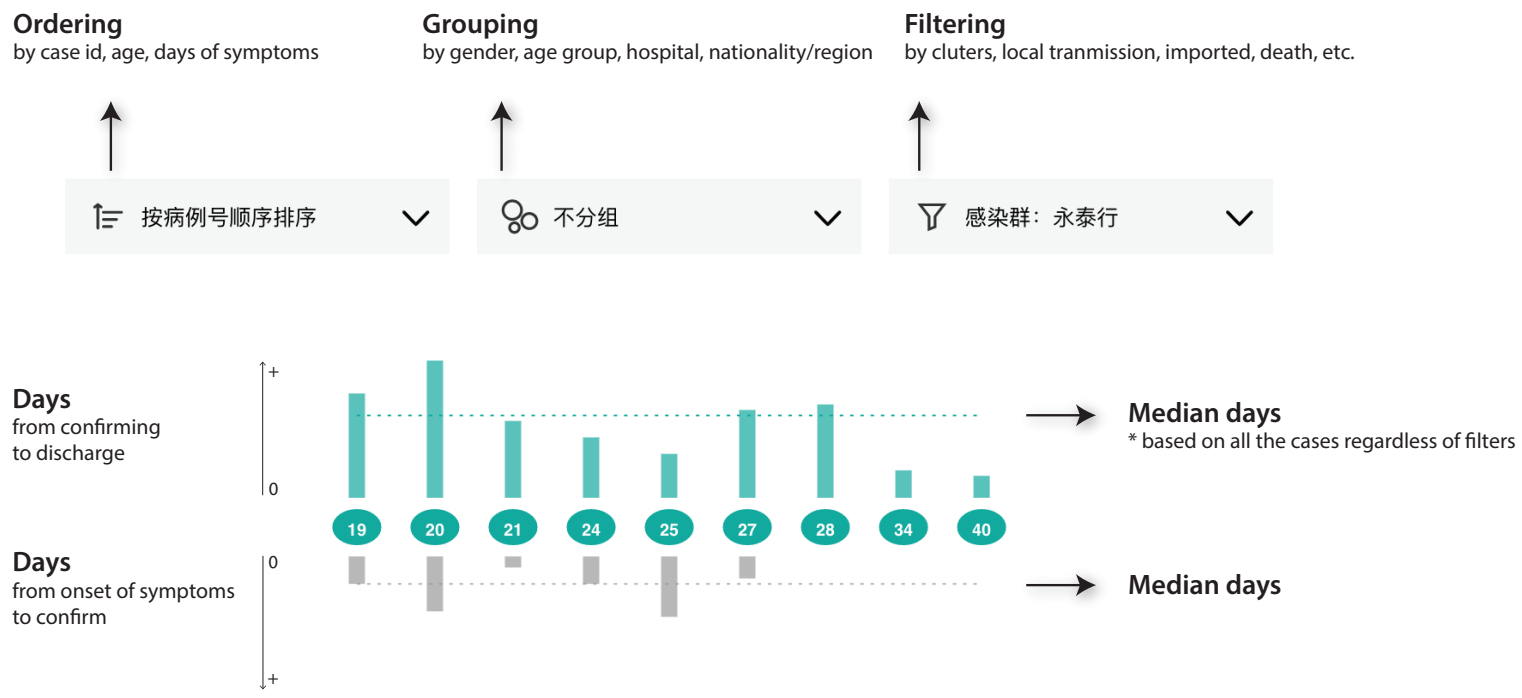

Case status death $\bigcirc$ discharged $\bigcirc$ hospitalized

Example: filtered by death cases, grouped by nationality/region

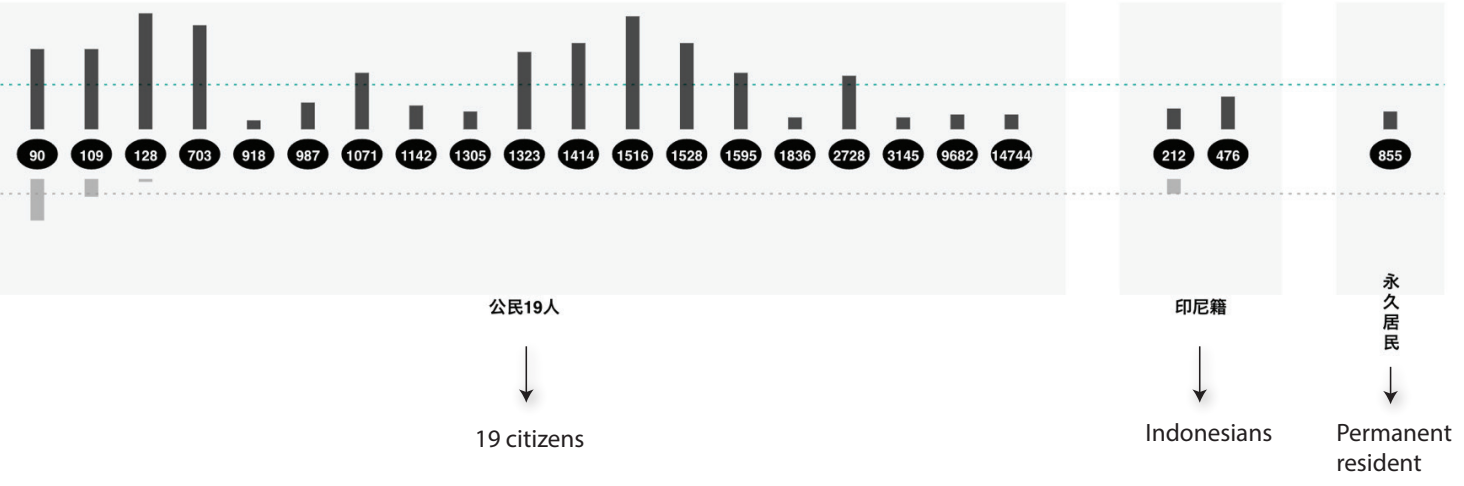


4: Visualization of discharged cases for summary

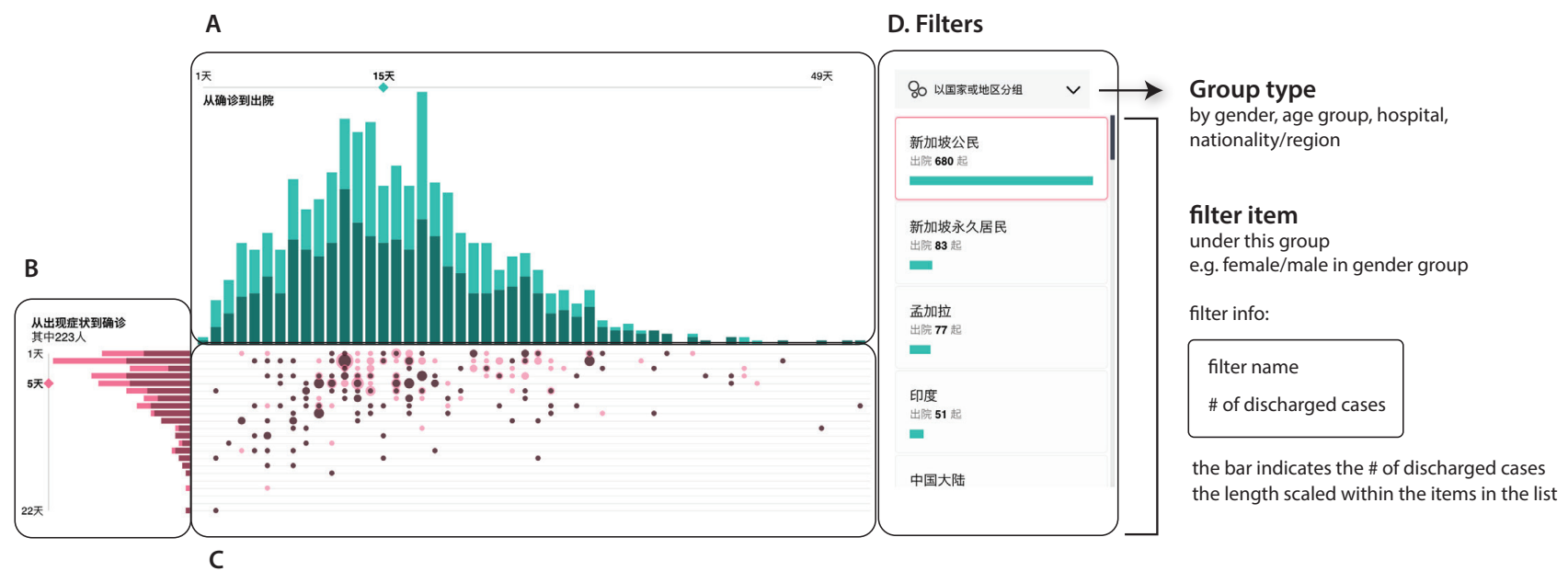

A. Distribution of hospitalization day days from confirming to discharge

$\checkmark$ median: 15 days total \# of cases f filtered \# of cases

B. Distribution of days of symptoms

days from onset of symptoms to confirm

*only 223 cases that have this data

median: 5 days total \# of cases

filtered \# of cases

\section{Distribution of the 223 cases that have both data}

total \# of cases filtered \# of cases larger size: larger \# of cases

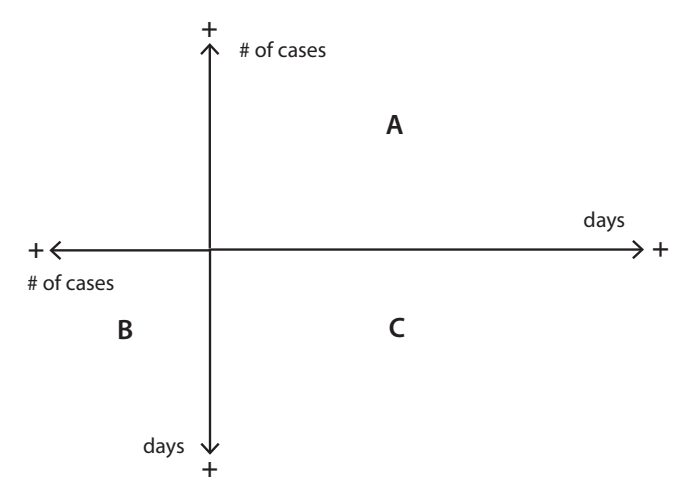

